\title{
Correlations between selected parameters of nasal cavity in neonates and young infants - computed tomography study
}

\author{
W. Likus ${ }^{1}$, K. Gruszczyńska², J. Markowski³ ${ }^{3}$ M. Machnikowska-Sokołowska², Z. Olczak4, \\ G. Bajor ${ }^{1}$, M.J. $\operatorname{tos}^{5,6}$, J. Baron ${ }^{7}$ \\ ${ }^{1}$ Department of Anatomy, School of Medicine, Medical University of Silesia, Katowice, Poland \\ ${ }^{2}$ Department of Radiodiagnostics and Invasive Radiology, School of Medicine, Medical University of Silesia, \\ Katowice, Poland \\ ${ }^{3}$ Department of Laryngology, School of Medicine, Medical University of Silesia, Katowice, Poland \\ ${ }^{4}$ Division of Diagnostic Imaging and Interventional Radiology, Upper Silesian Child's Health Centre, Katowice, Poland \\ ${ }^{5}$ Department of Clinical and Experimental Medicine, Division of Cell Biology Integrative Regenerative Med. Centre (IGEN), \\ Linköping University, Linköping, Sweden \\ ${ }^{6}$ Department of Pathology, Pomeranian Medical University, Szczecin, Poland \\ ${ }^{7}$ Department of Medical Imaging, School of Medicine, Medical University of Silesia, Katowice, Poland
}

[Received: 9 November 2015; Accepted: 13 November 2015]

Background: Correlations between selected metric parameters of the nasal cavity and nasopharynx in children without atresia may be useful for anticipating probable dimensions of this region in living subjects, in terms of changes with age. Materials and methods: One hundred and eighty children, age range $0-3$ years, were divided into five age groups, and measurements of 18 distances between structures of nasal cavity and nosopharynx were performed on computed tomography scans. Correlation coefficients and relations between parameters have been determined.

Results: Our study confirmed the existence of statistically significant correlations between linear dimensions within nasal cavity in children. The analysis demonstrated that for the values of following indexes: nasal septum length/piriform aperture width, and maximum length of the nasal septum/posterior nares width no statistically significant differences have been noted between age groups of children. All correlations have been positive. No statistically significant differences have been noted between the maximum width of the vomer and osseous parameters measured, both in the anterior and posterior part of the nasal cavity, and nasal septum length.

Conclusions: The size of posterior nares changed with age in children by a constant value. So far, no such an analysis has been carried out assessing potential correlations between linear dimensions for the entire nasal cavity, nasopharynx, length of the nasal septum in children, as well as proportions of individual linear dimensions of the anatomical structures analysed, in various age groups. (Folia Morphol 2016; 75, 3: 334-340)

Key words: correlations, nasal cavity, dimensions, infants, children, computed tomography 


\section{INTRODUCTION}

For the assessment of biology of structure development, particularly the dependencies between linear dimensions, various statistical methods can be applied [11]. In order to compare variables (groups of variables) and find out whether they differ from each other, tests for significance of differences between two or many variables are applied. However, if we want to find out the correlations between features, namely whether linear increase in a specific dimension is associated with proportional increase or decreases in other dimension, correlation analysis should be used as a statistical tool [11, 15]. In medicine, correlation analysis is a tool which allows assessing how a given variable, e.g. dimensions of the skull, dimensions of brain fluid space, or dimensions of posterior nares, change in dependence on another variable, e.g. age. Studies by various authors demonstrated that the width of posterior nares itself increases with age by an accurately defined value. This has been confirmed by our earlier studies, in which we demonstrated that this dimension increases at the rate of $0.162 \mathrm{~mm} /$ week [7]. Slovis et al. [14] assessed the change in posterior nares dimension in a group of neonates, children and young adults, from their day of birth until the age of 20. They demonstrated that the dimension increased by $0.027 \mathrm{~cm} /$ year. Sweeney et al. [16] assessed this parameter in patients of the age range between 2 and 13 years. The linear dependence increased with age and amounted to $0.208 \pm$ $\pm 0.09 \mathrm{~mm} /$ year. According to Corsten et al. [3], the dependence between increment of posterior nares and age amounted to $0.04 \mathrm{~mm} /$ week.

Because the first 3 years of age are a period of very intense growth of the organism and substantial percentage increase of body length, the authors divided the study group into five narrow age ranges, in order to check how wide and how many age ranges there should be, with separate norms for the parameters analysed.

In the literature published so far, the assessment of linear dimensions of bone structures of the nasal cavity, with the application of computed tomography (CT) scans, referred only and solely to posterior nares. On the other hand, there are no studies which would analyse the degree in which variables, namely nasal cavity dimensions in anterior and posterior part, together with nasopharynx dimension in transverse plane and nasal septum length would be correlated.

In the course of the study, the authors performed measurement of bone structures on CT scans, with layer width of $0.5 \mathrm{~mm}$. This is justified, as CT is presently the golden standard in diagnostics of nasal cavity diseases, thus it can be applied successfully as a tool for assessing anatomical structures [13]. Thin layers allow performing secondary multiplanar reconstructions and making the results obtained highly accurate. Additionally, the assessment of mutual dependencies between linear dimensions allows determining, whether the growth of individual bone structures is uniform, which of them grow faster and which grow slower, and that - in turn - determines growth trends in dependence on child age, and growth rate. The results obtained by the authors provide relevant information concerning the aspects of nasal cavity development.

The aim of our study was to determine the mutual relations between linear dimensions within the nasal cavity and nasopharynx in children without atresia (problems with patency of the nose), from birth to $3^{\text {rd }}$ year of age. The secondary aim of the study was to compare the analysed dimensions in five narrow age groups. The tertiary aim of the authors has been to calculate the length relations of selected linear dimensions and check whether they differ between the narrow age groups analysed.

\section{MATERIALS AND METHODS}

This retrospective study was approved by the Bioethics Commission of Medical University of Silesia.

\section{Materials}

Our analysis comprised CT scans of 180 Polish Caucasian children (83 girls and 97 boys), with regular development of the brain, without any craniofacial abnormalities, the age range of whom was from birth to 3 years of age. All the patients were examined in the Department of Radiodiagnostics and Invasive Radiology, Medical University of Silesia, Katowice, Poland.

The subjects who qualified for morphometric analyses were children who were referred to head $\mathrm{CT}$ examination due to suspected head injury. Excluded from the study were: children born preterm, with alterations within the bones of skull, with genetic disorders (e.g. Down's syndrome), mental retardation, congenital defects and/or complexes of congenital defects (e.g. CHARGE association, VATER association, Apert syndrome, Crouzon syndrome, Pfeiffer syndrome), craniosynostosis, and hydrocephalus. 
Only those tomograms have been included in the assessment of nasal cavity dimensions in which no deviations have been found from the standard condition of skull osseous structures.

\section{CT examinations}

Spiral technique, in transverse plane, in layers of $0.5 \mathrm{~mm}$ in thickness, using 64 row Toshiba Aquilion apparatus (Toshiba, Tokyo, Japan), in accordance with the standard diagnostic protocol for head examination. The obtained axial images from $\mathrm{CT}$ were transferred to a workstation for analysis. The measurement plane was parallel to the so-called Frankfurt plane (auriculo-orbital plane). The measurements were performed on scans at the level of nasal fossa floor, or just above it. In order to precisely visualise the air ducts, colour inversion has been applied. All measurements were performed directly on CT films, each measurement was taken with the accuracy of $0.01 \mathrm{~mm}$, the measurements were standardised to a $5-\mathrm{cm}$ reference scale on each film. The average of two separate measurements was used for analysis.

\section{Measurements (Fig. 1)}

In posterior nasal cavity we measured bony choanal aperture width between both pterygoid processes - choanal aperture (BCAW), right posterior bony width between bone sidewall and septal mucosa (RPBW), left posterior bony width between bone sidewall and septal mucosa (LPBW), length of septum between piriform aperture to the end of the vomer (LS), maximal length of septum between the most anterior part of nasal septum to the end of the vomer (MLS), maximal septum width (MSW), maximal width of the vomer (MVW), nosopharynx vertical distance between posterior vomer and cranial base (NVD), anterior bony width between the two ridges extruding from the maxilla - piriform aperture (ABW), right anterior bony width from the right maxillary ridge to the septal mucosa (RABW), left anterior bony width from the left maxillary ridge to the septal mucosa (LABW).

\section{Study group}

The study group has been divided into five age categories:

- A: 0-3 months (23 children: 13 females and 10 males);

- B: 4-6 months (35 children: 20 females and 15 males);
- C: 7-12 months (53 children: 27 females and 26 males);

- D: under 2 years of age (13-24 months) (42 children: 14 females and 28 males);

- E: under 3 years of age (25-36 months) (27 children: 9 females and 18 males).

\section{Data analysis}

Dependencies between bone parameters within the nasal cavity and the strength of these dependencies have been assessed by means of Pearson's correlation coefficient. The assessment of the correlation coefficient value has been quoted after Stanisz [15].

With the growth of a child's body, proportions between dimensions of specific body parts change. Those changes are most prominent within the head. That is why the authors determined the nasal cavity size ratios, comparing the dimensions at the level of the piriform aperture with dimensions of the posterior nares, as well as with the dimensions of nasal septum and nasopharynx width.

For the purpose of making comparisons between groups, the ANOVA test has been used, as post-hoc test the Tukey HSD test has been utilised. Statistical significance has been determined at the level of $p \leq 0.05$ and the analysis was carried out using STATISTICA software version 10.0 (StatSoft).

\section{RESULTS}

The measurements of the spaces between bone structure of nasal cavity, nasal septum and nasopharynx in newborns and young children have been summarised in Tables 1 and 2.

On the basis of average values of linear parameters of the nasal cavity in children, the authors made the attempt to determine the ratios that would describe dependencies in nasal cavity dimensions. For that purpose, ratios have been determined for bone dimensions, as well as chondro-osseous dimensions (septum) in the nasal cavity, in respective age groups. The values of ratios have been presented in Table 3 . The analysis revealed that for the LS/ABW, MLS/ABW, and MLS/BCAW ratios; no statistically significant differences have been noted in specific age groups of children. Those ratios for the entire age group under 3 years of age amount to: $2.05,2.83$, and 2.66, respectively. LS/ABW and LS/BCAW ratios have the same value in children below the age of 3 . Likewise, no statistically significant differences have been noted for the MLS/ABW and MLS/BCAW ratios. Analysing the 

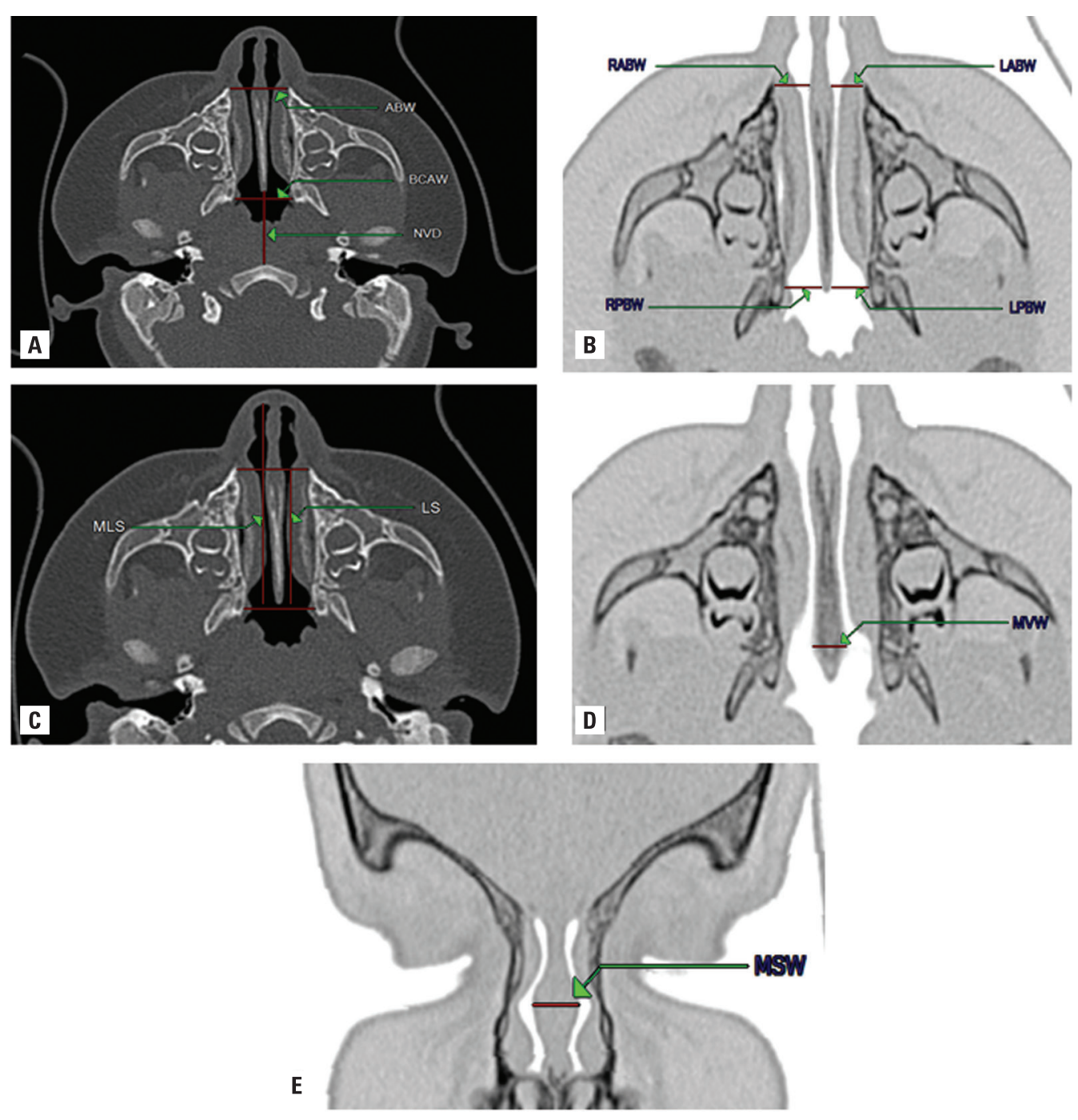

Figure 1. A-E. Normal nasal cavity — axial computed tomography image (child 4 month old). Abbreviations — see text.

Table 1. Dimensions of anterior and posterior nasal cavity

\begin{tabular}{lccccccc}
\hline Patients' & \multicolumn{3}{c}{ Anterior } & & \multicolumn{3}{c}{ Posterior } \\
\cline { 2 - 4 } \cline { 6 - 8 } groups & ABW & RABW & LABW & & BCAW & RPBW & LPBW \\
& {$[\mathrm{mm}]$} & {$[\mathrm{mm}]$} & {$[\mathrm{mm}]$} & & {$[\mathrm{mm}]$} & {$[\mathrm{mm}]$} & {$[\mathrm{mm}]$} \\
\hline Total & $\mathbf{1 7 . 1 4} \pm$ & $\mathbf{7 . 0 0} \pm$ & $\mathbf{6 . 6 5} \pm$ & & $\mathbf{1 8 . 2 4} \pm$ & $\mathbf{7 . 8 1} \pm$ & $\mathbf{7 . 7 3} \pm$ \\
& $\mathbf{1 . 3 3}$ & $\mathbf{1 . 1 1}$ & $\mathbf{1 . 0 2}$ & & $\mathbf{2 . 0 7}$ & $\mathbf{0 . 7 9}$ & $\mathbf{0 . 8 5}$ \\
$\mathrm{A}$ & $14.87 \pm$ & $6.26 \pm$ & $5.64 \pm$ & $16.86 \pm$ & $6.84 \pm$ & $6.62 \pm$ \\
& 1.62 & 1.11 & 1.04 & 2.16 & 0.88 & 0.85 \\
$\mathrm{~B}$ & $15.60 \pm$ & $6.29 \pm$ & $5.99 \pm$ & $16.70 \pm$ & $7.12 \pm$ & $6.92 \pm$ \\
& 1.15 & 0.99 & 0.81 & 1.26 & 0.63 & 0.81 \\
$\mathrm{C}$ & $17.11 \pm$ & $7.04 \pm$ & $6.60 \pm$ & $17.77 \pm$ & $7.58 \pm$ & $7.65 \pm$ \\
& 1.27 & 0.98 & 0.89 & 1.45 & 0.69 & 0.89 \\
$\mathrm{D}$ & $18.46 \pm$ & $7.43 \pm$ & $7.10 \pm$ & $19.26 \pm$ & $8.37 \pm$ & $8.35 \pm$ \\
& 1.35 & 0.92 & 1.06 & & 1.47 & 0.85 & 0.62 \\
E & $18.87 \pm$ & $7.71 \pm$ & $7.65 \pm$ & 20.65 & $9.00 \pm$ & $8.85 \pm$ \\
& 1.28 & 0.93 & 1.09 & \pm 1.53 & 0.90 & 0.67 \\
\hline
\end{tabular}

A: 0-3 months; B: 4-6 months; C: 7-12 months; D: under 2 years of age (13-24 months), and E: under 3 years of age (25-36 months); remaining abbreviations - see text
Table 2. Dimensions of nasal septum, vomer and nasopharynx

\begin{tabular}{|c|c|c|c|c|c|}
\hline \multirow{2}{*}{$\begin{array}{l}\text { Patients' } \\
\text { groups }\end{array}$} & \multicolumn{3}{|c|}{ Nasal septum } & \multirow{2}{*}{$\begin{array}{l}\text { Vomer } \\
\text { MVW } \\
{[\mathrm{mm}]} \\
\end{array}$} & \multirow{2}{*}{$\begin{array}{c}\text { Nosopharynx } \\
\text { NVD } \\
{[\mathrm{mm}]}\end{array}$} \\
\hline & $\begin{array}{c}\text { LS } \\
{[\mathrm{mm}]}\end{array}$ & $\begin{array}{l}\text { MLS } \\
{[\mathrm{mm}]}\end{array}$ & $\begin{array}{l}\text { MSW } \\
{\left[\text { [mm] }^{*}\right.}\end{array}$ & & \\
\hline Total & $\begin{array}{c}35.16 \pm \\
3.46\end{array}$ & $\begin{array}{c}48.45 \pm \\
5.03\end{array}$ & $\begin{array}{c}8.90 \pm \\
1.33\end{array}$ & $\begin{array}{c}3.79 \pm \\
0.88\end{array}$ & $\begin{array}{c}24.96 \pm \\
3.20\end{array}$ \\
\hline A & $\begin{array}{c}30.10 \pm \\
4.60\end{array}$ & $\begin{array}{c}41.55 \pm \\
6.40\end{array}$ & $\begin{array}{c}7.00 \pm \\
1.48\end{array}$ & $\begin{array}{c}3.57 \pm \\
1.00\end{array}$ & $\begin{array}{c}21.06 \pm \\
3.48\end{array}$ \\
\hline B & $\begin{array}{c}31.90 \pm \\
3.24\end{array}$ & $\begin{array}{c}43.67 \pm \\
3.67\end{array}$ & $\begin{array}{c}8.52 \pm \\
1.12\end{array}$ & $\begin{array}{c}4.03 \pm \\
0.74\end{array}$ & $\begin{array}{c}24.86 \pm \\
2.66\end{array}$ \\
\hline C & $\begin{array}{c}34.86 \pm \\
3.69\end{array}$ & $\begin{array}{c}48.22 \pm \\
6.14\end{array}$ & $\begin{array}{c}9.34 \pm \\
1.03\end{array}$ & $\begin{array}{c}3.84 \pm \\
0.95\end{array}$ & $\begin{array}{c}25.60 \pm \\
2.67\end{array}$ \\
\hline$D$ & $\begin{array}{c}38.28 \pm \\
2.84\end{array}$ & $\begin{array}{c}52.74 \pm \\
4.54\end{array}$ & $\begin{array}{c}9.10 \pm \\
0.93\end{array}$ & $\begin{array}{c}3.64 \pm \\
0.76\end{array}$ & $\begin{array}{c}25.62 \pm \\
2.40\end{array}$ \\
\hline$E$ & $\begin{array}{c}38.16 \pm \\
2.91 \\
\end{array}$ & $\begin{array}{c}53.97 \pm \\
4.44\end{array}$ & $\begin{array}{c}9.73 \pm \\
0.93 \\
\end{array}$ & $\begin{array}{c}3.82 \pm \\
0.98 \\
\end{array}$ & $\begin{array}{c}26.18 \pm \\
3.27\end{array}$ \\
\hline
\end{tabular}

*Measurement in coronal plane; A: 0-3 months; B: 4-6 months; C: 7-12 months; D: under 2 years of age (13-24 months), and $\mathbf{E}$ : under 3 years of age (25-36 months); remaining abbreviations - see text 
Table 3. Values of nasal cavity dimension ratios in respective age groups in children under three years of age

\begin{tabular}{lccccccccc}
\hline Patient & \multicolumn{10}{c}{ Ratios of selected parameters } \\
\cline { 2 - 11 } groups & ABW/BCAW & ABW/NVD & BCAW/NVD & LS/ABW & MLS/ABW & LS/BCAW & MLS/BCAW & LS/NVD & MLS/NVD \\
\hline Total & $\mathbf{0 . 9 4}$ & $\mathbf{0 . 9 6}$ & $\mathbf{0 . 7 3}$ & $\mathbf{2 . 0 5}$ & $\mathbf{2 . 8 3}$ & $\mathbf{2 . 0 5}$ & $\mathbf{2 . 6 6}$ & $\mathbf{1 . 4 1}$ & $\mathbf{1 . 9 4}$ \\
A & 0.88 & 0.72 & 0.82 & 2.03 & 2.79 & 1.80 & 2.79 & 1.49 & 1.99 \\
B & 0.94 & 0.63 & 0.67 & 2.05 & 2.81 & 1.92 & 2.81 & 1.30 & 1.78 \\
C & 0.97 & 0.67 & 0.70 & 2.05 & 2.89 & 1.97 & 2.89 & 1.36 & 1.90 \\
D & 2.96 & 0.73 & 0.76 & 2.12 & 2.86 & 2.03 & 2.86 & 1.53 & 2.09 \\
E & 0.92 & 0.73 & 0.80 & 2.02 & 2.86 & 1.85 & 2.86 & 1.48 & 2.09 \\
\hline
\end{tabular}

A: 0-3 months; B: 4-6 months; C: 7-12 months; D: under 2 years of age (13-24 months), and E: under 3 years of age (25-36 months); remaining abbreviations - see text

Table 4. Correlations between linear parameters of bones within the nasal cavity, in children between 0 and 3 years of age $(n=180)$

\begin{tabular}{|c|c|c|}
\hline \multirow[t]{2}{*}{ Correlation coefficient, $r_{\mathrm{xy}}$} & \multicolumn{2}{|c|}{ Relationships between measured structures } \\
\hline & Positive correlation & Negative correlation \\
\hline $\begin{array}{l}\text { High correlation } \\
\left(0.7 \leq r_{x y}<0.9\right)\end{array}$ & $\begin{array}{l}\text { ABW } \Leftrightarrow R A B W, L A B W, M L S \\
L P B W \Leftrightarrow R P B W \\
B C A W \Leftrightarrow R P B W, L P B W \\
L S \Leftrightarrow M L S\end{array}$ & - \\
\hline $\begin{array}{l}\text { Moderate correlation } \\
\left(0.5 \leq \mathrm{r}_{\mathrm{xy}}<0.7\right)\end{array}$ & $\begin{array}{l}A B W \Leftrightarrow B C A W, R P B W, L P B W, L S, M S W \\
R A B W \Leftrightarrow M L S \\
L A B W \Leftrightarrow M L S \\
B C A W \Leftrightarrow M L S \\
L S \Leftrightarrow L P B W \\
M L S \Leftrightarrow L P B W, R P B W\end{array}$ & - \\
\hline $\begin{array}{l}\text { Low correlation } \\
\left(0.3 \leq r_{x y}<0.5\right)\end{array}$ & $\begin{array}{l}A B W \Leftrightarrow N V D \\
R A B W \Leftrightarrow L A B W, B C A W, R P B W, \text { LPBW, LS, MSW } \\
L A B W \Leftrightarrow B C A W, R P B W, \text { LPBW, LS, NVD, MSW } \\
B C A W \Leftrightarrow L P B W \\
\text { RPBW } \Leftrightarrow L S, N V D \\
M S W \Leftrightarrow B C A W, \text { RPBW, LPBW, LS, MLS }\end{array}$ & - \\
\hline $\begin{array}{l}\text { Negligible correlation } \\
\left(0.1 \leq \mathrm{r}_{\mathrm{xy}}<0.3\right)\end{array}$ & NVD $\Leftrightarrow R A B W, B C A W$, LPBW, LS, MLS, MSW & - \\
\hline $\begin{array}{l}\text { No correlation } \\
\left(r_{x y}=0\right)\end{array}$ & 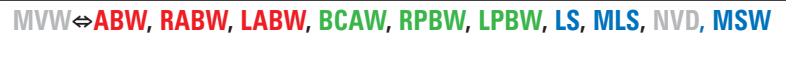 & \\
\hline
\end{tabular}

piriform aperture; choanae; nasal septum; nasopharynx vertical distance

statistically significant differences in ratio values in the age groups $A-E$, differences have been found to exist for the following age groups: $A$ vs. $B$ for ratios BCAW/NVW ( $p<0.01)$, LS/NVW ( $p=0.041)$; A vs. C for ratios ABW/BCAW ( $p<0.001)$, BCAW/NVW $(p<0.05)$; A vs. D for ratios ABW/BCAW $(p<0.05)$ and LS/BCAW ( $p=0.032$ ); B vs. $D$ and $B$ vs. $E$ for ratios $A B W / N V W(p<0.0001, p<0.01)$, BCAW/NVV $(p<0.01, p<0.001)$ and LS/NVW $(p<0.001, p=0.035)$; $C$ vs. D for the ratio ABW/NVW $(p<0.05)$, and C vs. E for the ratio BCAW/NVW ( $<<0.01$ ). No statistically significant differences have been noted to exist between groups: A vs. E and D vs. E.

The authors, when analysing the dependencies between morphometric parameters of the nasal cav- ity and child's age, regardless of the child's sex, have found that dimensions increase with age, as regards all linear parameters of bone structures (Table 4). The linear correlation coefficient had its highest values for the parameter in the anterior part of the nasal cavity - the bony distance at the level of nasal notch (incisura nasalis) (ABW) amounting to $r_{x y}=0.734$, and the parameter defining the distance between left bone sidewall and septal mucosa (LPBW), amounting to $r_{x y}=0.701$. We have noted increasing width of posterior nares in children under 3 years of age; the increase rate was $0.162 \mathrm{~mm} /$ month. No statistically significant correlation has been noted to exist between vomer width and age of the child $(p=0.710)$. A high level of positive correlation, statistically significant, has 
been demonstrated to exist between the child's age on the one hand and length of chondro-osseous nasal septum (LS), $r_{x y}=0,639$, and maximum length of the nasal septum (MLS), $r_{x y}=0.691$, on the other hand. For nasopharynx vertical dimension (NVD) we have noted an average positive correlation of $r_{x y}=0.351$, statistically significant. Our previous study confirmed the correlation between nasal cavity dimensions and child's age, yet that correlation was of different strength [7]. In connection with the above, assessment has been made of mutual dependencies between the analysed structures, and the strength of those dependencies has been assessed, taking into account the location of measured dimensions within the nasal cavity and nasopharynx.

The increase in bony distance at the level of the maxilla nasal notches (ABW) results in the increase in: distance between maxilla nasal notch and septal mucosa on the right and left side (RABW, LABW) that are located at the level of the piriform aperture, bony width of posterior nares (BCAW), posterior bony width between bone sidewall and septal mucosa on the right and left (RPBW, LPBW) in the posterior part of the nasal cavity. The values of relations strength are provided in Table 4. The existence of average positive correlation has been found between bony width of posterior nares (BCAW) and maximum width of nasal septum (MSW). Analysing the nasopharynx vertical dimension (NVD), a dependence was noted, namely that when that dimension (width) increases, the bony choanal aperture width (BCAW) increases, as well as the length of chondro-osseous nasal septum (LS) and maximum septum length (MLS). However, the described correlations concerning nasopharynx dimensions were weak.

No statistically significant dependencies have been noted between the MVW and bony parameters measured both in the anterior and posterior part of nasal cavity, as well as nasal septum length.

\section{DISCUSSION}

Upper respiratory tract, which includes the nasal cavity, consists of structures that are important for conduction of air to the lungs. Development begins from early stages of intrauterine life. The post-natal development of upper respiratory tract is strictly connected with the development of the skull skeleton [10]. During the first 2-3 years of child's life, the development of that area accelerates [8]. CT is a good tool for assessing bone structures, including those in the nasal cavity [7, 12]. In accordance with the results of the study conducted by Weitzman et al. [18], in which measurements made on skulls were compared with the measurements of bone structures on CT images, minimum differences between direct measurements on skulls and indirect measurements on tomograms confirm the supposition that measurements made on CT images are credible and accurately reflect the scanned object. The analysed structures are located deep within bony face and their morphometry - in intravital conditions - may be examined only by means of imaging methods, of which CT is the best. That is why the authors used CT scans as a tool for determining the size of bony structures in the area, in order to show the dependencies between growing nasal cavity bone structures in children below 3 years of age. To obtain better precision and accuracy of measurements, they have been made on CT scans with layer width of merely $0.5 \mathrm{~mm}$.

In order to assess the mutual linear dependencies between two different variables, correlation analysis is a useful tool, as well as determination of correlation coefficient $\left(r_{x y}\right)$ [17]. According to Mukaka [9], and Bland and Altman [2] the relationships identified using correlation coefficients should be interpreted as associations but not as casual relationships.

The study reported here is the first of that type, which shows the results of analysis of the dependencies in linear growth of nasal cavity and nasopharynx structures in children, from their birth to the age of 3 . So far, the strength and direction of mutual relations between nasal cavity dimensions and the width of nasopharynx have not been assessed in children or youngsters. The authors of the study attempted, for the first time, to assess the correlations for those structures. Analysing the results we obtained, one should take into account the dynamics of growth of the structures within the facial skeleton in children, including the nasal cavity. The studies of Djupesland et al. [5] revealed that the size of air space in the nasal cavity does not depend on the sex, but is in positive correlation - having statistical significance - with the size of body, to be exact with the head size. Thus, in the study reported here, the authors assessed the dependence between variables in age groups, regardless the sex of the child. One has to keep in mind, when analysing the results obtained, the dynamics of changes in nasal cavity dimensions in children. The total minimum surface area of nasal cavity cross-section in the first year of life, measured with the use of rhinomanometry, increases by $67 \%$ 
(from $0.21 \mathrm{~cm}^{2}$ to $0.35 \mathrm{~cm}^{2}$ ), its size increases by nearly a half $[4,5]$. On the other hand, the volume of the upper part of the nasal cavity increases by $36 \%$ [4]. At the age of 7 , the nasal cavity is twice higher [4]. Also the proportion of its height to width changes: in a newborn it is $1: 1$; in a child under 3 years of age it is 3:2 [4]. Analysing the correlation coefficient, which reflects the strength of relation between variables, we demonstrated that the air space of posterior nares undergoes changes in the first 3 years of life. The assessment of mutual dependencies between structures, particularly bone structures, may be made by means of ratios/indexes. Clinically recognised indexes which define the dependencies between bone structures are: cranial index [6] or mandibular canine index used in orthodontics [1]. The analysis of the indicators we determined showed that for LS/ABW, MLS/ABW, and MLS/BCAW ratios no statistically significant differences have been noted between values in particular age groups of children. Analysing the correlations between age groups, we have not detected negative correlations - having statistical significance - between linear measurements analysed in children under 3 years of age. Correlations of high level have been noted mainly for dimensions of the piriform aperture and nasal septum. No statistically significant dependencies have been registered between MVW and bone parameters measured, both in the anterior and posterior part of the nasal cavity or the length of nasal septum.

\section{CONCLUSIONS}

Postnatal development of upper respiratory tract is strictly connected with the development of child's head skeleton. Despite the fact that the period between birth and the age of 3 is when - proportionally - the growth process is the fastest - our study revealed that the development of particular bone structures in nasal cavity in children, that we measured, is not uniform, which has been indicated by different values of correlation indicators. The differences in the growth rate of specific bone structures of the nasal cavity have been reflected in different values of dimension relations for the bone structures analysed. Those differences indicate that as early as then, the process of newborn skull shape change begins, including also the nasal cavity.

\section{REFERENCES}

1. Bakkannavar SM, Manjunath S, Nayah VC, Kumar P (2014) Canine Index: a tool for sex determination. Egyptian J Forensic Sci, 5: 157-161. doi: 10.1016/j.ejfs.2014.08.008.

2. Bland JM, Altman DG (1995) Calculating correlation coefficients with repeated observations: Part 2. Correlation between subjects. BMJ, 310:633.

3. Corsten MJ, Bernard PA, Udjus K, Walker R (1996) Nasal fossa dimensions in normal and nasally obstructed neonates and infants: preliminary study. Int J Pediatr, Otorhinolaryngol, 36: 23-30.

4. Crelin ES (1973) Functional anatomy of the newborn. Yale University Press, New Haven and London.

5. Djupesland PG, Lyholm B (1998) Changes in nasal dimensions in infancy. Acta Otolaryngol, 118: 852-858.

6. Likus W, Bajor G, Gruszczyńska K, Baron J, Markowski J, Machnikowska-Sokołowska M, Milka D, Lepich T (2014) Cephalic index in the first three years of life: study of children with normal brain development based on computed tomography. Scientific World J, 2014: 502836.

7. Likus W, Bajor G, Gruszczyńska K, Baron J, Markowski J (2014) Nasal region dimensions in children: a CT study and clinical implications. Biomed Res Int, 2014: 125810.

8. Moore KL, Persaud TVN, Torchia, MG (2011) The developing human: clinically oriented embryology. 9th Ed. Saunders, Philadelphia.

9. Mukaka MM (2012) Statistics corner: a guide to appropriate use of correlation coefficient in medical research. Malawi Med J, 24: 69-71.

10. Pohunek P (2004) Development, structure and function of the upper airways. Paediatr Respir Rev, 5: 2-8.

11. Riffenburgh RH (2012) Statistics in medicine. $3^{\text {rd }}$ Ed. Elsevier Inc.

12. Rysz M, Bakoń L (2009) Maxillary sinus anatomy variation and nasal cavity width: structural computed tomography imaging. Folia Morphol, 68: 260-264.

13. Skóra W, Kozłowski Z, Dabrowska K, Mazerant M (2008) Clinical evaluation of CT scans of nasal cavity and paranasal sinuses. Otolaryngol Pol, 62: 553-557.

14. Slovis TL, Renfro B, Watts FB, Kuhns LR, Belenky W, Spoylar J (1985) Choanal atresia: precise CT evaluation. Radiology 155: 345-348.

15. Stanisz A (2007) Przystępny kurs statystyki w oparciu o program STATISTICA PL na przykładach z medycyny. StatSoft Polska, Kraków [in Polish].

16. Sweeney KD, Deskin RW, Hokanson JA, Thompson CP, Yoo JK (1997) Establishment of normal values of nasal choanal size in children: comparison of nasal choanal size in children with and without symptoms of nasal obstruction. Int J Pediatr Otorhinolaryngol, 39: 51-57.

17. Taylor R (1990) Interpretation of correlation coefficient: a basic review. JDMS, 1: 35-39.

18. Waitzman AA, Posnick JC, Armstrong DC, Pron GE (1992) Craniofacial skeletal measurements based on computed tomography: Part I. Accuracy and reproducibility. Cleft Palate Craniofac J, 29: 112-117. 